\title{
Sexual abuse of doctors by doctors: professionalism, complexity and the potential for healing
}

\author{
Sexual abuse in the medical profession \\ is a complex, multifaceted problem that \\ needs evidence-based solutions
}

\begin{abstract}
$\mathrm{C}$ ontemporary attitudes to sexual abuse are changing. The Royal Commission into Institutional Responses to Child Sexual Abuse, the response of the Australian Defence Force to allegations of sexual abuse in the military and the work of the Australian Human Rights Commission around sexual harassment in the workplace all indicate a shift in community values. They also represent a shift in our understanding of the nature and scope of professionalism. As each respected institution has its professional failures exposed, it becomes obvious that no group is immune. Existing codes of professional conduct have not protected colleagues or clients from toxic behaviour.
\end{abstract}

\section{Sexual abuse in medicine}

The recent discussions in the mainstream and social media have sparked national and international attention, on both the allegations of entrenched sexual harassment, misogyny and exploitation within the surgical profession, and the institutional response to these claims. Essentially, the medical profession has claimed that existing policies protect junior doctors by encouraging victims to report inappropriate behaviour. However, this perspective fails to recognise the profound power imbalance that exists between senior and junior staff. Given the personal and professional cost of whistleblowing, ${ }^{1}$ it is understandable that victims choose to remain silent.

There is little written about the sexual abuse of qualified doctors in the workplace, but there is extensive literature detailing pervasive bullying and harassment of medical students. The literature is complicated by inconsistent definitions, with inappropriate behaviour occurring on a continuum. Sexual harassment covers a broader range of unwanted behaviours than sexual assault or sexual violence. A recent systematic review and metaanalysis of harassment and discrimination suggests that $59.4 \%$ of medical trainees had experienced harassment or discrimination during their training. ${ }^{2}$ Consultants were the most commonly cited offenders. The authors concluded that the average prevalence of sexual harassment was $33 \% .^{2}$ Other studies report a higher incidence among women. ${ }^{3,4}$

The impact of this abuse is profound. In a 1990s study, $69 \%$ of those abused reported that the abuse was of "major importance and very upsetting". ${ }^{5}$ Half (49.6\%) of the students indicated that the most serious episode "the "hidden curriculum' may be more powerful than professionalism training"

(sti
struggle to manage the unprofessional behaviour of
supervisors. ${ }^{9}$ They described feelings of guilt and were resigned to the fact that these events would affect their personal and professional identity. ${ }^{9}$ It also seems that doctors and nurses have different expectations. While nurses tend to challenge abusive behaviour, medical students tend to acquiesce. ${ }^{10}$

Disturbingly, remedial efforts by institutions to prevent sexual harassment appear ineffective. On completion of a 10-year program to prevent medical student mistreatment in a United States hospital, the problem persisted with little change in prevalence. ${ }^{11}$ The authors concluded that the "hidden curriculum" may be more powerful than professionalism training. ${ }^{11}$

\section{Changing our professional culture for the future}

It seems unlikely that sexual harassment ceases at graduation, so it is interesting that little is written about abuse within the medical profession. Without published literature, there can be little clinical or academic conversation about the management of the victims of abuse, and no evidence-based organisational response to the culture that enables or tolerates this behaviour. However, some levers for change already exist.

There is much to learn from the way professional expectations, policies and processes have shifted to manage other unwanted professional behaviours, such as medical errors. It has been necessary to tackle the tendency to deny errors, to cover up the evidence, and doi: 10.5694/mja15.00378 to avoid frank and open discussion. ${ }^{12}$ This shift has 
been difficult and has required a corresponding shift in cultural norms. It has involved research at all levels, including cognitive studies of clinical reasoning and organisational research into institutional behaviour.

Preventing and managing sexual harassment and sexual assault will require a similar breadth of research and policy change. At the organisation level, the Australian Medical Council accreditation framework requires all Colleges to meet Standard 7.4 on the management of bullying and harassment. The 2014 survey of doctors in training by the Australian Medical Association (AMA) found that $30 \%$ of participants felt their College had clear and accessible policies on bullying and harassment, and only $12 \%$ felt that their College responded in a timely manner to such complaints. ${ }^{13}$ In light of the AMA's findings and recent events, it appears that this standard and assessment against it need urgent review. ${ }^{14}$ Similarly, National Safety and Quality Health Service Standards could strengthen their emphasis on the safety and security of staff. ${ }^{15}$

To understand and modify professional culture, however, we also need to explore the experience of individuals in the context of hierarchical medical teams, and follow the impact of abuse over time. Specifically, further work should critically examine the cultural frameworks that enable abuse and promote the silence of victims, and should explore the way doctors seek help and support.

To help promote healing, we need to produce and adopt an empirically derived framework for developing therapeutic guidelines that explicitly redress the personal and professional impact of sexual harassment and sexual assault in the medical profession.

Qualitative research into the experience of young doctors who experience abuse in the workplace would be the first step in understanding these complex behaviours, and would be the first study of its type internationally. Collaborative research is necessary to expose, question and correct toxic cultural practices, and to create safe working environments for all doctors in the future.

Sexual harassment and sexual assault are illegal, deeply traumatic and profoundly unprofessional. However, given the ongoing harassment of medical students internationally, it is likely that this abuse is an entrenched part of medical culture. The cause is not simple, but we must heal ourselves. It is high time the profession critically examined this problem from multiple perspectives and provided a multifaceted, committed and evidence-based approach to changing this toxic culture.

Competing interests: No relevant disclosures.

Provenance: Not commissioned; externally peer reviewed. 
1 McDonald S, Ahern K. The professional consequences of whistleblowing by nurses. J Prof Nurs 2000; 16: 313-321.

2 Fnais N, Soobiah C, Chen MH, et al. Harassment and discrimination in medical training: a systematic review and meta-analysis. Acad Med 2014; 89: 817-827.

3 Rademakers JJ, Van Den Muijsenbergh ME, Slappendel G, et al. Sexual harassment during clinical clerkships in Dutch medical schools. Med Educ 2008; 42: 452-458.

4 Nora LM, McLaughlin MA, Fosson SE, et al. Gender discrimination and sexual harassment in medical education: perspectives gained by a 14-school study. Acad Med 2002; 77: 1226-1234.

5 Silver HK, Glicken A. Medical student abuse: incidence, severity, and significance. JAMA 1990; 263: 527-532.

6 Lubitz RM, Nguyen DD. Medical student abuse during third-year clerkships. JAMA 1996; 275: 414-416.

7 Richardson DA, Becker M, Frank RR, Sokol RJ. Assessing medical students' perceptions of mistreatment in their second and third years. Acad Med 1997; 72: 728-730.

8 Richman JA, Flaherty JA, Rospenda KM, Christensen ML. Mental health consequences and correlates of reported medical student abuse. JAMA 1992; 267: 692-694.

9 Babaria P, Abedin S, Berg D, Nunez-Smith M. "I'm too used to it": a longitudinal qualitative study of third year female medical students' experiences of gendered encounters in medical education. Soc Sci Med 2012; 74: 1013-1020.
10 Timm A. "It would not be tolerated in any other profession except medicine": survey reporting on undergraduates' exposure to bullying and harassment in their first placement year. BMJ Open 2014; 4: e005140. doi: 10.1136/bmjopen-2014-005140.

11 Fried JM, Vermillion M, Parker $\mathrm{NH}$, Uijtdehaage S. Eradicating medical student mistreatment: a longitudinal study of one institution's efforts. Acad Med 2012; 87: 1191-1198.

12 Patient Safety Unit/Health Systems and Services Cluster, World Health Organization African Region. Guide for developing national patient safety policy and strategic plan. Brazzaville: WHO Regional Office for Africa, 2014. http://www.who.int/patientsafety/guide-for-developing-nationalpatient-safety-policy-and-strategic-plan_final.pdf (accessed Jul 2015).

13 Australian Medical Association. 2014 AMA Specialist trainee survey: report of findings. Canberra: AMA, 2015. https://ama.com.au/sites/default/files/ documents/2014_AMA_Specialist_Trainee_Survey_Report_of_Findings_0. pdf (accessed Jul 2015).

14 Australian Medical Council. Standards for assessment and accreditation of specialist medical education programs and professional development programs by the Australian Medical Council 2010. Canberra: AMC, 2010. http://www.amc.org.au/joomla-files/images/Accreditation/standardsfor-specialist-medical-training-2010.pdf (accessed Jul 2015).

15 Australian Commission on Quality and Safety in Health Care. Standard 1. Governance for safety and quality in health service organisations. Safety and quality improvement guide. Sydney: ACOSHC, 2012. http://www. safetyandquality.gov.au/wp-content/uploads/2012/10/Standardl_ Oct_2012_WEBl.pdf (accessed Jul 2015). 